\title{
Implant-supported cantilevered fixed partial dentures
}

\author{
Abstracted from \\ Zurdo j, Romao C, Wennstrom JL. \\ Survival and complication rates of implant-supported fixed partial dentures with cantilevers: a systematic review. \\ Clin Oral Implant Res 2009; 20 (suppl. 4): S59-S66 \\ Address for correspondence: José Zurdo, Institute for Postgraduate Dental Education, \\ Greenbank Building, Room 304, University of Central Lancashire, Preston, Lancashire, UK. \\ E-mail: jose.zurdo@btopenworld.com
}

\section{Question: How do the survival and complication rates of implant-supported fixed partial dentures with cantilevers compare with implanted-supported single tooth and implanted-supported fixed partial dentures without cantilevers?}

Data sources Medline was used to search for relevant material for the review.

Study selection Systematic reviews and longitudinal prospective/ retrospective studies (randomised controlled trials, controlled clinical trials and cohort studies) were chosen that reported outcomes following treatment with implant-supported fixed partial denture prostheses (FPDP) with cantilever extensions, after a mean function time of at least 5 years. Two independent reviewers preformed screening and data abstraction.

Data extraction and synthesis Five-year survival and technical complication rates were extracted, which included: loss of prosthesis, loss of implants, complications with supra-construction (fractures or deformations of the framework or veneers, loss of retention and screw or abutment loosening) and marginal bone loss. Data from included studies were pooled and summarised as a weighted mean. Disagreement regarding data extraction was resolved by discussion and consensus.

Results Only three studies met the inclusion criteria for final analysis. Two of the studies had a prospective or retrospective case-control design, whereas the third was a prospective cohort study. The 5-year survival rate of cantilever FPDP varied between 89.9 and $92.7 \%$ (weighted mean, 91.9\%), with implant fracture as the main cause for failure. The corresponding survival rate for FPDP without cantilever extensions was 95.3 to $96.2 \%$ (weighted mean, 95.8\%). Technical complications related to the supra-construction in the three included studies were reported to occur at a frequency of 13-26\% (weighted mean, 20.3\%) for cantilever FPDP, compared with 0-12\% (weighted mean, 9.7\%) for noncantilever FPDP. The most common complications were minor porcelain fractures and bridge-screw loosening. For cantilever FPDP, the 5-year event-free survival rate varied between 66.7 and $79.2 \%$ (weighted mean, $71.7 \%$ ) and between 83.1 and 96.3\% (weighted mean, 85.9\%) for noncantilever FPDP. No statistically significant differences were reported with regard to peri-implant bone-level change between the two prosthetic groups, either at the prosthesis or at the implant level.
Conclusions Data on implant-supported FPDP with cantilever extensions are limited and therefore survival and complication rates should be interpreted with caution. The incorporation of cantilevers into implant-borne prostheses may be associated with a higher incidence of minor technical complications

\section{Commentary}

The introduction of dental implants to clinical practice has increased the available prosthetic treatment options for the partially edentulous. This review by Zurdo et al. compares the clinical performance between implant-supported FPDP with and without a cantilever extension, after at least 5 years of use. In this study, the latter group also included the implant-supported single tooth prosthesis. ${ }^{1}$ A summary of the review findings (in the form of 5-year weighted means) is presented in Table 1 .

I agree with this article's discussion that, “... the result of this study should be interpreted with caution because of the small sample size." Also threatening the validity of this review is how data were aggregated to calculate a summary statistic (weighted mean) from three very different study designs (ie, there was heterogeneity in study design). For example, included in this review was an article by Halg et al, ${ }^{2}$ which was a case-control study where only nine out of

\begin{tabular}{|c|c|c|}
\hline & $\begin{array}{l}\text { Cantilever } \\
\text { [mean }(95 \% \mathrm{CI})]\end{array}$ & $\begin{array}{l}\text { Noncantilever } \\
\text { [mean }(95 \% \mathrm{CI})]\end{array}$ \\
\hline \multicolumn{3}{|l|}{ Survival (\%) } \\
\hline Prosthesis* & $91.9(95.8-88.0)$ & $4.2(96.3-95.3)$ \\
\hline Implants $^{\dagger}$ & $91.9(88.0-95.6)$ & $95.8(95.3-96.3)$ \\
\hline $\begin{array}{l}\text { Technical } \\
\text { complications }(\%)^{\ddagger}\end{array}$ & $21.6(11.9-31.2)$ & $10.3(2.1-18.5)$ \\
\hline Boneless (mm) & $0.35(0.15-0.41)$ & $0.14(0.04-0.32)$ \\
\hline Event-free (\%) & $71.7(64.2-79.1)$ & $85.9(77.7-94.1)$ \\
\hline \multicolumn{3}{|l|}{$\mathrm{Cl}$, Confidence interval. } \\
\hline \multicolumn{3}{|c|}{$\begin{array}{l}\text { *Total number of cantilever and noncantilever prosthesis are } 74 \text { and } 142 \text { respectively. } \\
\text { Calculated from data given in article. }\end{array}$} \\
\hline \multicolumn{3}{|c|}{$\begin{array}{l}\text { TTotal number of implants in the cantilever and non-cantilever groups are } 178 \text { and } \\
247 \text { respectively. Calculated from data given in article. }\end{array}$} \\
\hline
\end{tabular}


the 27 case-subjects and 15 out of the 27 control subjects included were observed for 5 years or longer. Halg et al. still used a weighted mean failure rate, however, which included all 27 case and 27 control subjects. In turn, Zurdo and colleagues, in this review, then used this flawed estimate within their own weighted mean calculations. ${ }^{1}$

Wennstromm, one of the authors of this review, included one of his own studies. ${ }^{3}$ The aim of this included study was to retrospectively analyse whether the inclusion of cantilever extensions increased the amount of marginal bone loss with freestanding, implant-supported, FPDP over a 5-year period of functional loading. Only 45 out of the original 51 subjects were included in the final analysis. Three subjects were lost to followup and the other three "experienced implant failures". ${ }^{3}$ These censored data were not accounted for in the final analysis. Furthermore, it is not clear from this original study why the three patients with implant failure were not included. This could negatively affect the reported failure rate.

A Kaplan-Meier analysis is typically used in studies that observe time-to-event outcomes because it accounts for censored data, thus giving a more accurate statistic of the outcome. ${ }^{4}$ Only the Kreissl et al. article included in this review carried out such analysis. ${ }^{5}$ The purpose of that prospective study was to evaluate the incidence of the most common technical problems, namely screw loosening, screw fracture, fracturing of veneering porcelain and framework fracture in implant-supported FPDP, and to assess the survival and success rate (event-free survival) after 5 years. Only 51 out of the original 76 subjects, however, were still being observed after 60 months. It is not clear what happened to the other 25 subjects. Were they lost to followup or did they have their appliances inserted fewer than 5 years ago?

Typically, weighted means, as presented in the Zurdo et al. systemic review, are fraught with errors that can lead to spurious conclusions. A comparative survival analysis between two treatment options is typically presented as a hazard ratio. This ratio is the relative risk of survival between two treatment options through the time-to-event period under investigation. The hazard ratio accounts for censored data, therefore making it suitable for inferential statistical analysis. This review could not offer a valid meta-analysis of the hazard ratios without having access to the individual patient data from each study. Such a process is rigorous and requires some similarity in how outcomes are measured in the included studies. Considering the significant heterogeneity between the three included here, it is unlikely that a valid meta-analysis of the hazard ratios could be carried out.

Even if we take the authors' summary weighted-mean statistic at face value (Table 1), the noncantilever group also included the implant-supported single tooth crown. This design is not an alternative to the multiple tooth prosthesis for which a cantilever bridge would be indicated. Therefore, in this review, the outcomes of the noncantilever group are biased by the well-established and high success rate of the implant-supported single tooth implant. ${ }^{6}$ Furthermore, the cantilever implant-supported bridge is often an alternative to the edentulous space being restored, with an implant abutment on one end and a natural tooth abutment on the other. Such a design was not included in this review.

All that being said, this review does add to our current knowledgebase the observation that the implant-supported cantilever fixed bridge appears to give high success rates. Further data are needed to confirm or deny whether their survival and complication rates are as impressive as the noncantilever implant-supported prosthesis, including the implant-supported single tooth crown.

Ben Balevi

Private practitioner, affiliated with Faculty of Medicine, University of British Columbia, Vancouver, British Columbia,

Canada

1. Zurdo j, Romao C, Wennstrom JL. Survival and complications rates of implantsupported fixed partial dentures with cantilevers: a systematic review. Clin Oral Implant Res 2009; 20 (suppl. 4): S59-S66.

2. Halg GA, Schmid J, Hammerle CH. Bone level change at implants support crowns or fixed partial dentures with and without cantilevers Clin Oral Implant Res 2008; 19: 983-990.

3. Wennstrom J, Zurdo J, Karlsson S, Ekestubbe A, Grondahl K, Lindhe J. Bone level changes at implant-supported fixed partial dentures with and without cantilever extensions after 5 years in function. J Clin Periodont 2004; 31: 1077-1083.

4. Chapman D. Modelling Survival Data in Medical Research. 2nd Edn. London: Chapman and Hall; 2003.

5. KriessI ME, Gerds T, Muche R, Heydecke G, Strub IR. Technical complications of implant supported fixed partial dentures in partially edentulous cases after an average observation period of 5 years Clin Oral Implant Res 2007; 18: 720-726.

6. Bader J. Implant supported single crowns offer good 5-year survival, but with complications. J Evid Based Dent Pract 2008; 8: 232-233.

Evidence-Based Dentistry (2010) 11, 48-49. doi:10.1038/sj.ebd.6400721 